\title{
A Comparison of Different Training Methods in the Successful Learning of Endobronchial Ultrasound-Guided Transbronchial Needle Aspiration
}

\author{
Wen Chao Zhang ${ }^{a} \quad$ Wei Chen ${ }^{a-c}$ Jian Ping Zhou ${ }^{a, b} \quad$ Andrew D. Lerner $^{c}$ \\ Lei Ni $^{a}$ Ji Min Shen ${ }^{a}$ b Tian Li Yan ${ }^{b}$ Min Zhou $^{a}$ Guo Chao Shi ${ }^{a}$ b Yi Xiang ${ }^{a-c}$ \\ ${ }^{a}$ Department of Respiratory Medicine and ${ }^{\mathrm{b}}$ Interventional Pulmonology Medical Center, RuiJin Hospital, \\ Shanghai Jiao Tong University, School of Medicine, Shanghai, PR China; 'Department of Interventional \\ Pulmonology, Division of Pulmonary and Critical Care Medicine, Johns Hopkins University School of \\ Medicine, Baltimore, MD, USA
}

\section{Keywords}

Training methods · Learning curve · Cumulative sum analysis · Lung cancer - Endobronchial ultrasound-guided transbronchial needle aspiration

\begin{abstract}
Background: Standardized training of endobronchial ultrasound-guided transbronchial needle aspiration (EBUSTBNA) is available only in highly developed countries. Objective: To compare the skill differences in the performance of EBUS-TBNA by pulmonologists trained under different learning methods. Methods: We performed a retrospective study of 145 EBUS-TBNA cases. We set up 3 comparative training groups: a standardized training group, a teaching group, and a self-study group. The main clinical outcomes were sensitivity, specificity and accuracy of EBUS-TBNA. Cumulative sum analysis was used to track EBUS-TBNA proficiency of the 3 groups. Results: The sensitivities of EBUSTBNA in the different groups were 90,88 , and $67 \%$, respectively. The accuracies of the different groups were 91, 90,
\end{abstract}

(c) 2017 S. Karger AG, Basel and $71 \%$, respectively. With respect to the sensitivity and accuracy, there was no significant difference between the standardized training group and teaching group. However, the self-study group was inferior to the other 2 groups. By comparing the learning curve among the groups, we found that the minimum number of operations needed to reach a $90 \%$ accuracy for the standardized training group, the teaching group and the self-study group was 17, 32 and 40 cases, respectively. Conclusion: Compared with the teaching and self-study groups, the standardized training group resulted in practitioners achieving high skill levels following the performance of a lower number of cases. Pulmonologists trained in the teaching group achieved a similar level of skills, but required more experience to reach this level. Practitioners in the self-study group did not achieve a satisfactory level of skills, despite performing a high number of cases.

(c) 2017 S. Karger AG, Basel

W.C. Zhang, W. Chen and J.P. Zhou contributed equally to this work.

\section{KARGER}

E-Mail karger@karger.com

www.karger.com/res
Yi Xiang

Department of Respiratory Medicine, RuiJin Hospita Shanghai Jiao Tong University, School of Medicine

Building 36, 7th Floors, Shanghai 200025 (PR China)

E-Mail xiangyiht@163.com 


\section{Introduction}

Over the past decade, endobronchial ultrasoundguided transbronchial needle aspiration (EBUS-TBNA) has been proven to be a valuable method for evaluating mediastinal and hilar lymphadenopathy as well as other lesions. This minimally invasive technique was first developed during the early 1990s, and became widely available in 2005. So far, it has been recommended as a first-line procedure for the diagnosis and staging of lung cancer by the official guideline of the European Society of Gastrointestinal Endoscopy (ESGE), produced in cooperation with the European Respiratory Society (ERS) and the European Society of Thoracic Surgeons (ESTS) as well as the American College of Chest Physicians (ACCP) and National Comprehensive Cancer Network (NCCN) guidelines [1-4]. Compared with the conventional transbronchial needle aspiration (C-TBNA) and surgical staging alone, EBUS-TBNA has been found to be superior in performing effective and secure biopsies of intrathoracic structures [5-8]. Individually, EBUSTBNA had the highest diagnostic yield of sarcoidosis (74.5\%), which was better than C-TBNA [5]. Endosonography (followed by surgical staging if negative) had higher sensitivity (SEN) and negative predictive values (NPV), resulted in fewer unnecessary thoracotomies and better quality of life during staging, and was slightly more effective and less expensive than surgical staging alone [8].

Since its development, many specialized hospitals and endoscopy centers have introduced EBUS bronchoscopy into their clinical practice. Numerous clinicians have started to learn EBUS-TBNA [9]. However, compared with the C-TBNA, the complexity of this advanced diagnostic procedure can make mastering this procedure a difficult and time-consuming process [10]. In order to improve the efficiency of clinical diagnosis and to reduce the incidence of complications, operators need to have a good understanding of the EBUS bronchoscopy equipment, the EBUS-TBNA procedure technique, the anatomy of the mediastinum, and its related sonographic landmarks. Therefore, a high degree of training and experience is necessary to achieve an acceptable level of proficiency.

In 2003, the ACCP recommended a minimum of $40-$ 50 procedures be performed for radial EBUS to achieve competency, but these were expert-based and preceded the introduction of the linear EBUS-TBNA [11]. As such, there are no established guidelines for a threshold number of procedures needed for competency in linear EBUS-
TBNA and controversy remains [12]. Along with constant practice, formal training courses exist, many of which are run by interventional pulmonary training programs, and recommended for the novice. Unfortunately, the numbers of such courses are limited in availability, typically found only in highly developed countries. In addition, these courses tend not to be systematically approved and standardized by their respective educational organizations [13]. Therefore, only few physicians, especially in developing countries, have the opportunity to receive formal training.

Most of clinical physicians learn the technique of EBUS-TBNA through secondary teaching methods, usually from a combination of literature review, videos, and from practicing on inanimate models at "hands-on" courses. At present, there is limited data to support the best method of EBUS-TBNA learning, especially regarding training within a formal fellowship program compared with those learning outside of a formal program and relying on secondary measures.

In this study, we performed a retrospective study of EBUS-TBNA at the Shanghai Ruijin Hospital. 145 EBUSTBNA cases were reviewed to analyze the value of EBUSTBNA in the diagnosis of mediastinal lesions. By comparing the SEN, specificity, and accuracy (ACC) of EBUSTBNA among a standardized training group (ST group), a teaching group (T group), and a self-study group (SS group), we evaluated the differences in skill levels among these groups. This study is intended to help teaching medical centers establish the most efficient, proficient, and economical models for training novice physicians in the performance of EBUS-TBNA.

\section{Study Objective and Method}

Equipment and Technique

The convex probe EBUS apparatus used in the Shanghai Ruijin Hospital is a flexible bronchoscope with a $7.5-\mathrm{MHz}$ convex ultrasound transducer placed at the tip of the scope (XBF-UC260FOL8; Olympus, Tokyo, Japan). This linear curved array ultrasound transducer scans parallel to the insertion direction of a bronchoscope needle. The ultrasound image is processed in a dedicated ultrasound scanner (EUC2000; Olympus) and is visualized along with the conventional white light bronchoscopy image.

The team performing EBUS-TBNA at the Shanghai Ruijin Hospital Pulmonary Medicine Endoscopy Center consisted of 3 senior attending doctors (5-10 years of work experience as general pulmonologists) and 2 chief physicians (work experience as general pulmonologists exceeding 15 years) as well as 2 nurses. All of the operating members had long-term operating experience of ordinary bronchoscopy ranging from 6 to 14 years, before they began to learn EBUS-TBNA. Each EBUS-TBNA case was per- 
formed with 2 physicians and a nurse. One physician was responsible for the bronchoscope operation and puncture localization, and the other was responsible for puncturing and processing biopsy specimens. The nurse was responsible for ancillary work.

In our pulmonary medicine endoscopy center, the anesthesia method of all of the EBUS-TBNA operations was lidocaine local anesthesia and midazolam associated with morphine for sedation and analgesia.

\section{EBUS-TBNA Operation Group Members}

According to the framework of the study, the operators were divided into an ST group, a T group, and an SS group. We named the 5 physicians as doctors $\mathrm{A}, \mathrm{B}, \mathrm{C}, \mathrm{D}$, and $\mathrm{E}$, respectively, for the convenience of description.

The ST group was comprised of doctors A and B with basic bronchoscopy experience of 7 and 14 years, respectively. These 2 members completed a 2-month interventional pulmonary training program at the Department of Interventional Pulmonology, Johns Hopkins University, School of Medicine, Baltimore, MD, USA. Their training experience included formal training in the anatomy of the airway and mediastinal structures, related ultrasound landmarks, and lymph node localization. Training in the operational technique included review of related videos and observation of at least 50 clinical cases in the endoscopy center. At the same time, they practiced EBUS-TBNA on simulator models many times. They were given an official certificate of EBUS-TBNA from the Department of Interventional Pulmonology, Johns Hopkins Hospital after their training program.

When the 2 members of the ST group had operated 10 EBUSTBNA cases together and reached a high ACC, they instructed the other 3 physicians (doctors $\mathrm{C}, \mathrm{D}$, and $\mathrm{E}$ ). The bronchoscopy experiences of these 3 pulmonary physicians were 12,8 , and 6 years. The 3 pulmonary physicians had first learned the anatomy of the airway and mediastinal structures, and the operational technique of EBUS-TBNA through literature and videos; then, they were instructed in the essentials of the instrument and operation by the ST group members. Later, they had received at least 5 EBUSTBNA clinical demonstrations in the endoscopy center.

The T group (doctors $A+C$ or doctors $B+C$ ) is composed of 1 ST group member and a fixed person (doctor C). The T group underwent direct supervision by the ST group member in the performance of clinical EBUS-TBNA cases.

The SS group was comprised of doctors D and $\mathrm{E}$ who underwent no formal procedural supervision, but performed EBUSTBNA only after review of the literature, videos, and 5 EBUSTBNA case demonstrations alone.

\section{General Information of Patients}

All patients with known or suspected lung cancer undergoing EBUS-TBNA for tissue diagnosis or staging between July 2011 and August 2015 in the Shanghai Ruijin Hospital pulmonary medicine endoscopy center were prospectively entered into a database. A total of 145 EBUS-TBNA cases were reviewed to analyze the value of EBUS-TBNA in the diagnosis of hilar and mediastinal lesions. Within the study group of 145 cases, the mean patient age was $55.30 \pm 11.09$ years, and 99 out of the 145 participants $(68 \%)$ were men. The numbers of EBUS-TBNA cases operated by the ST group, the T group, and the SS group were 36,63 , and 46 , respectively. All of the EBUS-TBNA operations have got the informed consent of the patients.

A Comparison of Training Methods in the Successful Learning of EBUS-TBNA

\section{Analysis Method}

Definition of a Successful EBUS-TBNA Needle Operation

We consider an operation to have been successful if the realtime ultrasound imaging showed that the puncture needle had been inserted into the target lesion and that the specimen had been adequately sampled for pathological examination. Otherwise, the procedure was considered unsuccessful.

\section{Definition of a Positive EBUS-TBNA Result}

A positive EBUS-TBNA was defined as pathology results consistent with malignancy or benign nodal pathology. A negative result was defined as normal lymphoid findings or reactive lymphadenopathy. Furthermore, positive results were validated if they either matched the clinical suspicion of disease or, in cases where the pathology did not match clinical suspicion, further diagnostic steps (such as mediastinoscopy, transthoracic needle aspiration, peripheral lymph node or endobronchial biopsies) ruled out a false-positive result.

\section{Statistical Method}

All of the diagnostic results of EBUS-TBNA were compared with other procedures (such as mediastinoscopy and other sampling techniques) or with conclusive clinical and radiographic follow-up for a minimum of 6 months. This was used to calculate SEN, specificity (SPE), ACC, positive predictive value (PPV), and $\mathrm{NPV}$. Outcomes were further separated according to the different training groups. $p<0.05$ was considered statistically significant. All data were analyzed with a statistical software package (SPSS, version 21.0)

The learning curve of each operation group was depicted by the Origin software (Origin 9.1, OriginLab, USA). The cumulative sum (CUSUM) equation is defined as $\delta=\mathrm{Xi}-\mathrm{X} 0$. The CUSUM graphical trend is summarized as follows: $\mathrm{Si}=\sum(\mathrm{Xi}-\mathrm{X} 0)$, where $\mathrm{Xi}=0$ for reaching one's goal, and $\mathrm{Xi}=1$ for failing to reach one's goal; "i" represents the number attempted; and X0 = the acceptable failure rate [14]. The acceptable failure rate is usually set by consensus of the related medicine department. Individual CUSUM graphs were plotted for each operation group. The minimum operation cases needed for each group to reach a target proficiency of EBUS-TBNA was figured out by the CUSUM analysis.

\section{Results}

\section{Positive EBUS-TBNA Results}

Within the study group of 145 cases, a total of 91 cases received a diagnosis of malignancy (lung cancer $n=86$, breast cancer $n=2$, esophageal cancer $n=2$, and lymphoma $n=1$ ), whereas 36 cases received a diagnosis of benign pathology ( $a$ arcoidosis $n=25$, tuberculosis $n=2$, parasitic infection $n=1$, nontuberculosis or nonparasitic ["ordinary"] infection $n=8$ ), and 18 cases had a diagnosis of reactive lymph node enlargement. 
General Information about Lymph Node TBNA

Within the 145 cases included, 140 cases had successful EBUS-TBNA punctures. Of the 5 unsuccessful operation cases, 3 were not punctured in the objective lesion due to the close proximity to large vessels and concern for risk of massive bleeding and for 2 cases, no adequate sample was achieved despite puncture attempt. The total success rate of operation was $97 \%$ (140/145). Mediastinal lymph nodes in our study were grouped according to the lung cancer lymph node map proposed by the IASLC lung cancer staging project in 2009 [15]. The most frequent mediastinal lymph nodes punctured were level 7 lymph nodes $(n=78)$, and the $4 \mathrm{R}$ group $(n=56)$ was next, followed by the $4 \mathrm{~L}$ group $(n=28)$. Level $10 \mathrm{R}(n=5), 11 \mathrm{~L}$ $(n=5), 2(n=4), 11 \mathrm{R}(n=3), 12 \mathrm{R}(n=2), 10 \mathrm{~L}(n=1)$, and $12 \mathrm{~L}(n=1)$ lymph nodes were less punctured.

36 EBUS-TBNA cases were operated by the ST group. In the ST group, a total of 56 lymph nodes were successfully punctured under EBUS guidance in 34 successful operation cases. The success rate of operation in the ST group was $94 \%$ (34/36). The mean number of lymph nodes was $1.65(56 / 34)$. The maximum number of lymph nodes punctured per case was 4 .

63 cases were operated by the T group. In the T group, a total of 71 lymph nodes were successfully punctured in 61 successful operation cases. The success rate of operation in the T group was $97 \%(61 / 63)$. The mean number of lymph node sampled per case was 1.16 (71/61). The maximum number of lymph nodes punctured per case was 3.

46 cases were operated by the SS group. In the SS group, a total of 56 lymph nodes were successfully punctured under EBUS guidance in 45 successful operation cases. The success rate of operation in the SS group was $98 \%$ (45/46). The mean number of lymph node sampled per case was 1.24 (56/45). The operators of the SS group punctured at most 2 lymph nodes per case.

There were no significant differences in the success rate of operation among the 3 groups, and there was no significant difference in the mean number of lymph nodes sampled per case between the T group and the SS group $(\mathrm{T}>0.05)$. However, the mean number of lymph nodes punctured per case in the ST group was significantly higher than in the T group $(p<0.001)$ and the SS group $(p<0.01)$.

Several studies have found that the diameter of lymph nodes was a key factor in determining operation success, especially for lymph nodes greater than $2 \mathrm{~cm}$ in diameter [16]. In our study, the ratios of lymph nodes greater than $2 \mathrm{~cm}$ in diameter to those less than $2 \mathrm{~cm}$ in the ST group,
Table 1. The diagnostic efficiency of EBUS-TBNA

\begin{tabular}{llllll}
\hline & SEN & SPE & ACC & PPV & NPV \\
\hline ST group & $90 \%$ & $100 \%$ & $91 \%$ & $100 \%$ & $50 \%$ \\
T group & $88 \%$ & $95 \%$ & $90 \%$ & $97 \%$ & $79 \%$ \\
SS group & $67 \%$ & $83 \%$ & $71 \%$ & $92 \%$ & $48 \%$ \\
$p$ (ST vs. T group) & 0.96 & 1 & 0.84 & 1 & 0.3 \\
$p$ (ST vs. SS group) & 0.02 & 1 & 0.02 & 0.2 & 1 \\
$p$ (T vs. SS group) & 0.02 & 0.27 & 0.01 & 0.69 & 0.02 \\
\hline
\end{tabular}

EBUS-TBNA, endobronchialultrasound-guided transbronchial needle aspiration; SEN, sensitivity; SPE, specificity; ACC, accuracy; $\mathrm{PPV}$, positive predictive value; NPV, negative predictive value; ST group, standardized training group; T group, teaching group; SS group, self-study group.

the T group and the SS group were 43/13, 49/22, and $40 / 16$, respectively. There were no significant differences in lymph node size among the 3 groups.

In conclusion, the ST group successfully punctured more lymph nodes per case than the T group and the SS group, though there were no significant differences between the SS group and the T group. As for the success rate of operation and the size of lymph nodes, there was no significant difference among the 3 groups.

\section{The Diagnostic Efficiency of EBUS-TBNA}

We used SEN, SPE, ACC, PPV, and NPV to determine the diagnostic efficiency of EBUS-TBNA. Within the 140 patients operated successfully, positive and negative cases diagnosed by EBUS-TBNA were 89 and 51, respectively. Positive and negative cases diagnosed by other procedures (i.e., mediastinoscopy or other sampling procedures) or with conclusive clinical and radiographic follow-up for a minimum of 6 months were 105 and 35, respectively. From these data, the overall SEN, SPE, ACC, PPV, and NPV of EBUS-TBNA were 82, 91, 84, 97, and $63 \%$, respectively. We compared the diagnostic efficiency of EBUS-TBNA among groups by analysis of variance. The statistical results are provided in Table 1.

In terms of the diagnostic efficiency of EBUS-TBNA, there were no significant differences between the ST group and the T group $(p>0.05)$. The ST group and the T group had a higher SEN and ACC of EBUS-TBNA than the SS group $(p<0.05)$. In terms of SPE and PPV, there were no significant differences among the 3 groups. In terms of NPV, there was a significant difference between the T group and the SS group $(p<0.05)$.

In conclusion, the diagnostic efficiency of EBUSTBNA in the T group was the same as that in the ST 
Table 2. Incidence of complications

\begin{tabular}{llll}
\hline & $\begin{array}{l}\text { Intraoperative } \\
\text { complications }\end{array}$ & $\begin{array}{l}\text { Postoperative } \\
\text { complications }\end{array}$ & $\begin{array}{l}\text { Total } \\
\text { complications }\end{array}$ \\
\hline ST group & $1 / 36$ & $3 / 36$ & $4 / 36$ \\
T group & $6 / 63$ & $4 / 63$ & $10 / 63$ \\
SS group & $2 / 46$ & $4 / 46$ & $6 / 46$ \\
\hline
\end{tabular}

ST group, standardized training group; $\mathrm{T}$ group, teaching group; SS group, self-study group.

group. Both the ST and T groups were superior in SEN and ACC compared to the SS group.

\section{Incidence of Adverse Events}

Within the 145 cases included, the procedural complications included bleeding requiring intervention (5 cases), severe coughing (2 cases), and tachycardia (2 cases). The postoperative complications in $24 \mathrm{~h}$ include hemoptysis (5 cases) and fever (6 cases). There were no serious complications in the 145 EBUS-TBNA cases. The incidences of complications among the 3 groups showed no significant difference $(p>0.05)$. The statistical results are provided in Table 2.

\section{The Learning Curve of EBUS-TBNA}

CUSUM was applied to demonstrate proficiency of the 3 operation groups in EBUS-TBNA. The evaluation index used in this study to describe the learning curve of EBUSTBNA is the ACC of cases. The operator is expected to reach a target proficiency of $90 \%$ ACC of EBUS-TBNA. The CUSUM graphical trend is summarized as follows: $\mathrm{Si}=\sum(\mathrm{Xi}-\mathrm{X} 0)$. When an operation result from EBUSTBNA is true positive or true negative, the operation is considered successful and $\mathrm{Xi}=0$; otherwise, it will be considered a failure and $\mathrm{Xi}=1$. The acceptable failure rate is set at $10 \%$ by consensus of our hospital, so $\mathrm{X} 0=0.1$. We used the Origin software to curve fit the data. Individual CUSUM graphs were plotted for each operation group. The CUSUM of the 3 groups is provided in online supplementary Table S1 (for all online suppl. material, see www.karger.com/doi/10.1159/000462978). The learning curves of the 3 groups are shown in Figure 1.

Based on the function curve of each group, when $\mathrm{X}=$ $16.684,31.39$, and 39.587 , respectively, the slope of the each learning curve accordingly takes on 0 . So, we conclude that the minimum operation cases needed for the ST group, the T group, and the SS group to reach $90 \%$ ACC were 17, 32, and 40, respectively.

A Comparison of Training Methods in the Successful Learning of EBUS-TBNA

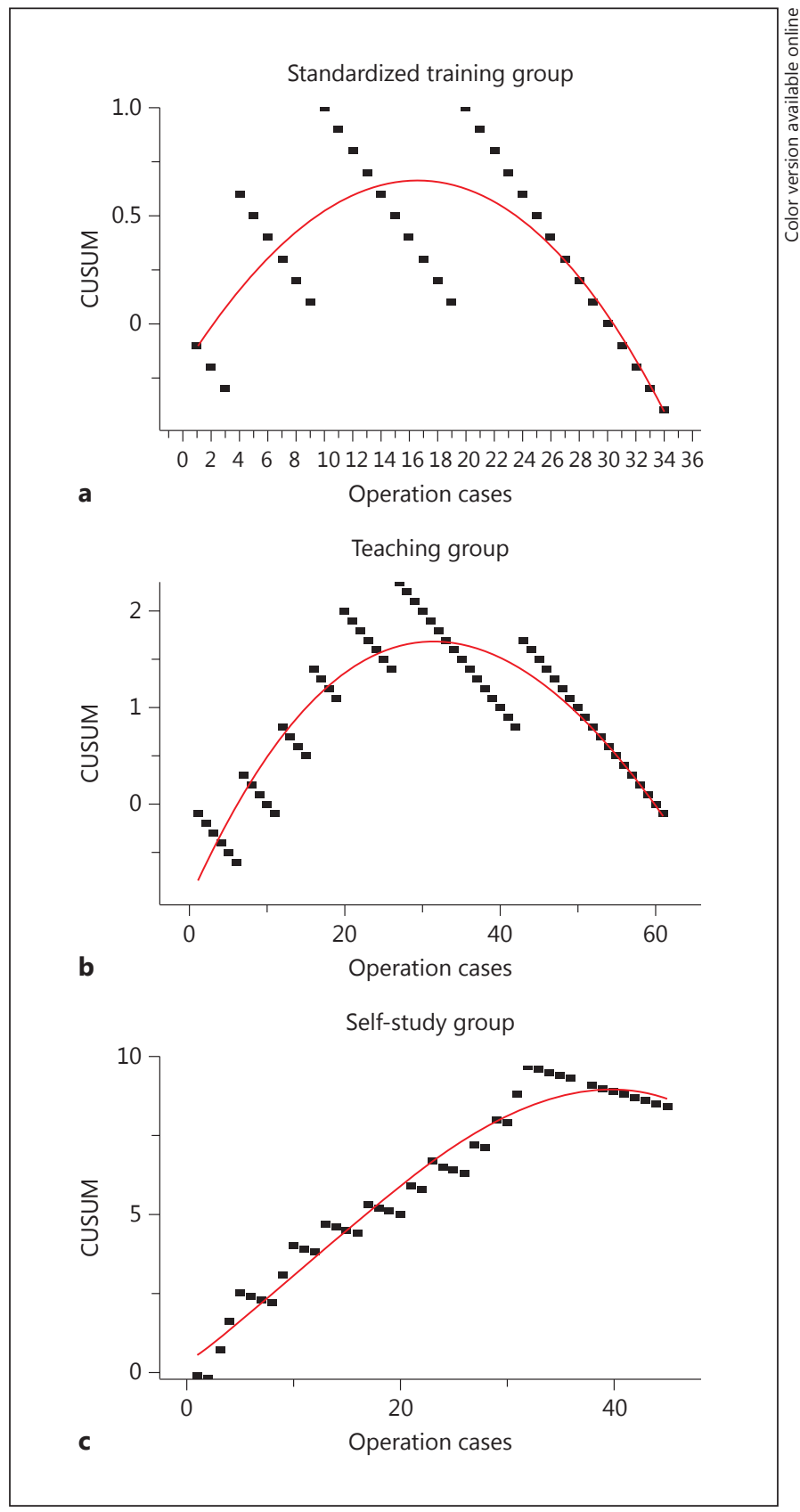

Fig. 1. The learning curves of different training groups. a Function curve of the standardized training group: $y=-0.19894+0.09996 \mathrm{x}$ $-0.00267 \mathrm{x}^{2}-1.30154 \times 10^{-5} \mathrm{x}^{3}$. When $\mathrm{X}=16.684$, the slope of the curve takes on 0 . So, the minimum operation cases needed for the standardized training group to reach $90 \%$ accuracy was 17. b Function curve of the teaching group: $\mathrm{y}=0.97345+0.17934 \mathrm{x}-0.00333 \mathrm{x}^{2}$ $+1.00534 \times 10^{-5} \mathrm{X}^{3}$. When $\mathrm{X}=31.39$, the slope of the curve takes on 0 . So, the minimum operation cases needed for the teaching group to reach $90 \%$ accuracy was 32 . c Function curve of the selfstudy group: $y=0.27749+0.24872 \mathrm{x}+0.0041 \mathrm{x}^{2}-1.2195 \times 10^{-5} \mathrm{x}^{3}$. When $\mathrm{X}=39.587$, the slope of the curve takes on 0 . So, the minimum operation cases needed for self-study group to reach $90 \%$ accuracy was 40 . 


\section{Discussion}

EBUS-TBNA is regarded as a safe and accurate diagnostic procedure for the diagnosis of mediastinal and hilar lymphadenopathy and other lesions within the thorax [17]. Since Yasufuku et al. [18] first reported its excellent SEN and SPE in 2006, many multicenter studies and meta-analyses have highlighted the diagnostic advantages of EBUS-TBNA [19, 20].

However, given the complexity of the EBUS equipment and of the EBUS-TBNA procedure itself, the operative difficulty of this relatively new technology is increased markedly compared with conventional TBNA [7]. Firstly, since the outer diameter of the EBUS scope is a little bigger than the conventional bronchoscope, the EBUS scope is typically inserted through the mouth rather than the nose. Consequently, operators who are accustomed to bronchoscopy performed transnasally may have difficulty adapting to the larger scope size. Secondly, given the 35-degree forward oblique view, passing the scope safely through the glottis and through the airways is often challenging for the novice and requires frequent practice. Thirdly, difficulty can arise in reliably obtaining a clear sonographic view of the lesion as the tip of the ultrasound probe must make direct contact with the airway mucosa. This may require skill in the adequate manipulation of the scope as well as knowledge in the use of an inflatable saline-filled balloon covering the probe itself. Finally, since conventional bronchoscopy is frequently performed together with EBUS bronchoscopy, the switching of the scopes during the procedure may increase levels of discomfort in patients under only moderate sedation. These reasons exemplify the specialized degree of training in order to master the skills of EBUS-TBNA.

The European Respiratory Society has made a formal EBUS training program in order to train qualified doctors to be able to independently and competently perform EBUS and ensure that participants have all the necessary knowledge and skills required to obtain ERS certification in EBUS. In the training program, organizers put great emphasis on simulator-based training. There are several studies; even a randomized controlled trial has reported that virtual reality simulator could be used to provide reliable and valid assessment of competence in EBUSTBNA and simulator-based training was shown to be more effective than traditional apprenticeship training [21-23]. Yet, as far as we know, the EBUS simulator is so expensive that few endoscopy centers in developing countries can afford to buy it. In addition, standard training of EBUS-TBNA can be time-consuming and costly.
Furthermore, most practitioners in developing countries do not have the opportunities to receive such formal training.

We shall ask the question: can we find a better training program that leads to significantly improved skills and efficiency same as the standard formal training, as well as decreasing investment in manpower, time and cost, as an interventional pulmonary fellowship program in EBUSTBNA?

Based on this question, our study compared the EBUSTBNA skill levels of different training groups: an ST group, a T group, and an SS group at the Shanghai Ruijin Hospital. Our results showed that diagnostic efficiency was not significantly different in the ST and T groups; however, both groups were superior in diagnostic ACC compared with the SS group. Among the 3 groups, there was no difference in the success rate of each individual operation. We conclude that there is no significant difference in the skill levels achieved by operators taught in an ST or T group, though they are superior to self-taught operators.

EBUS-TBNA has a low incidence of adverse effects. Cough, infection, and bleeding are the most common symptoms and they are usually mild. Pneumothorax, sustained hypoxia, hypotension, and respiratory failure may also occur, but are exceedingly rare [24-26]. The ACCP Quality Improvement Registry, Evaluation, and Education (AQuIRE) program included 1,317 patients and reported complication rates of bleeding requiring intervention $(0.2 \%)$, pneumothorax $(0.53 \%)$, significant airway injury $(0.1 \%)$, sustained hypoxia $(0.3 \%)$, hypotension $(0.1 \%)$, and respiratory failure $(0.23 \%)$. The overall $24-\mathrm{h}$ complication rate was $1.44 \%$ [27]. A systematic review and a meta-analysis (1,299 patients) in 2008 and 2009 both reported similarly low complication rates [28, 29]. In our study, there were no serious complications in the 145 EBUS-TBNA cases and the incidences of complications showed no difference among the 3 groups.

We performed CUSUM, which was first introduced by Page in 1954 based on the earlier work of Hurst. The basic step of this method consists of recursive calculations of calculating a cumulative sum of residuals and detecting changes of the statistical properties by identifying deviations from a reference state. Because of its simplicity, CUSUM has been widely applied as a statistical and graphical tool. It can be used to track the success and failure at a technical skill and examines trends over time. It can also be used to demonstrate proficiency in a newly learned technical skill or as a measure of quality assurance once a technical skill has been mastered. When compared against 
a standard, CUSUM provides an objective tool that uses the data gathered through log books to determine whether an operator has achieved competency in a particular skill $[30,31]$.

As the skill level of EBUS-TBNA operators improves, the learning curve depicted by CUSUM can be generally divided into 3 stages: the rising stage, the plateau stage, and the descent stage. In the rising stage of the learning curve, the operators usually cannot reach their goal, so the majority of $\mathrm{Xi}$ values equal 1 , and the $\delta$ values are positive. When the $\delta$ value of each case is added up, the CUSUM value will be positive, indicating an increasing trend. In the plateau stage of the learning curve, an increasing number of operators can reach their goal, so more and more Xi values equal 0 and the $\delta$ values are negative. When the $\delta$ value of each case is added up, the CUSUM value approaches 0 . This causes the learning curve to flatten. In the descent stage of the learning curve, the operators can usually reach their goal, so the majority of the $\mathrm{Xi}$ values equal 0 and the $\delta$ values are negative. When the $\delta$ value of each case is added up, the CUSUM will be negative, indicating a decreasing trend. The learning curve is then analyzed on the basis of its slope. When the slope of the learning curve becomes 0 , the corresponding value is the minimum number of operations necessary to succeed at this skill.

The learning curve is an indicator of the amount of experience required for operators to gain a predefined level of diagnostic ACC. In a sense, this indicates the amount of practice required to achieve proficiency. Our research utilized CUSUM to track the success and failure at EBUS-TBNA and examine these trends over time. By comparing the learning curve of the ST group, the $\mathrm{T}$ group and SS group, we found that the minimum operation cases needed to reach $90 \%$ ACC was 17,32 , and 40 , respectively. Prior literature has reported on the improved SEN, SPE, and learning curve of EBUS-TBNA following performance after a certain number of cases. AbuHijleh et al. [32] reported that the SEN of EBUS-TBNA would increase markedly between 25 and 50 cases. Hu et al. [16] reported that the minimum operation cases needed to reach $90 \%$ ACC was 22 . Bellinger et al. [33] reported that a minimum of $20-25$ procedures is needed to achieve acceptable yields. Our study showed similar results achieved by the ST and T groups.

One of the implications of our study shows that practitioners in developing countries, of whom the majority does not have access to standard training, can still achieve a satisfactory level of skill in EBUS-TBNA after undergoing strict learning about the anatomy of the airway and

A Comparison of Training Methods in the Successful Learning of EBUS-TBNA mediastinal structures, related ultrasound landmarks, lymph node localization, and the operational technique of EBUS-TBNA through literature and videos, combined with performance of more than 32 cases under the guidance of an already experienced operator. On the other hand, self-study practitioners without supervision take significantly longer to achieve a satisfactory level of diagnostic ACC.

The major limitations of our study include the retrospective design leading to selection bias, the small sample size within the groups, and the relegation to a single-center institution. Therefore, we must be careful in applying our results to other institutions with differing technical protocols. In addition, we defined the "standardized training" as a 3-month course at the Johns Hopkins University Hospital in the United States. However, a formal interventional pulmonology training program in the United States is typically 1-2 years in length, and often includes the performance of hundreds of EBUS bronchoscopies. In Europe, it also takes months to complete the formal EBUS training program made by ERS and get the ERS certification. Therefore, we can only compare the value of a 3-month program at the Johns Hopkins University with the other groups.

In addition, there is a lack of objective criteria like a simulator-based evaluation system to evaluate the skill levels of operators in developing countries, including China. So, the importance of our study is the aim to help guide institutions, especially in developing countries, identifying a threshold of informal training and developing efficient, secure, and economical teaching programs in the performance of EBUS-TBNA.

\section{Conclusion}

Standardized training is important to master the skills of EBUS-TBNA. Compared with informal training, standardized training resulted in practitioners achieving high skill levels following the performance of a lower number of cases. Practitioners within a teaching arm can also expect to reach similar levels of skills, but will require a greater amount of experience. Practitioners in the selfstudy learning pattern did not achieve a satisfactory level of skills, despite performing a high number of cases. The self-study of technical procedures must be discouraged. This study indicated that the hand-to-hand learning pattern might be the most efficient, proficient, and economical model for training novice physicians in the performance of EBUS-TBNA in developing countries.

Respiration 2017;93:319-326

DOI: $10.1159 / 000462978$ 


\section{Acknowledgements}

We thank Prof. Ko Pen Wang (Department of Interventional Pulmonology, Division of Pulmonary Medicine and Critical Care Medicine, Johns Hopkins University School of Medicine, Baltimore, MD, USA) for critically reviewing the manuscript and Yi Tao and Lian Dong (Department of Respiratory Medicine, RuiJin Hospital, Shanghai Jiao Tong University, School of Medicine, Shanghai, PR China) for secretarial assistance. This work was sup- ported, in whole or in part, by the Shanghai Jiao Tong University Program (AE604102) and the Shanghai Municipal Hospitals' Rising and Leading Technology Program (SHDC12015115).

\section{Financial Disclosure and Conflicts of Interest}

The authors have nothing to disclose with regard to commercial support.

\section{References}

1 Vilmann P, et al: Combined endobronchial and oesophageal endosonography for the diagnosis and staging of lung cancer. European Society of Gastrointestinal Endoscopy (ESGE) Guideline, in cooperation with the European Respiratory Society (ERS) and the European Society of Thoracic Surgeons (ESTS). Eur Respir J 2015;46:40-60.

2 Bade B, Furukawa B, Tanner NT: Convex probe endobronchial ultrasound. Semin Respir Crit Care Med 2014;35:636-644.

3 Rivera MP, Mehta AC, Wahidi MM: Establishing the diagnosis of lung cancer: Diagnosis and management of lung cancer, 3rd ed: American College of Chest Physicians evidence-based clinical practice guidelines. Chest 2013;143:e142S-e165S.

4 Silvestri GA, Gonzalez AV, Jantz MA, et al: Methods for staging non-small cell lung cancer: diagnosis and management of lung cancer, 3rd ed: American College of Chest Physicians evidence based clinical practice guidelines. Chest 2013;143:e211S-e250S.

5 Gupta D, et al: Endobronchial ultrasoundguided transbronchial needle aspiration vs conventional transbronchial needle aspiration in the diagnosis of sarcoidosis. Chest 2014; 146:547-556.

6 Oki M, et al: Prospective study of endobronchial ultrasound-guided transbronchial needle aspiration of lymph nodes versus transbronchial lung biopsy of lung tissue for diagnosis of sarcoidosis. J Thorac Cardiovasc Surg 2012;143:1324-1329.

7 Ye T, Hu H, Luo X, Chen H: The role of endobronchial ultrasound guided transbronchial needle aspiration (EBUS-TBNA) for qualitative diagnosis of mediastinal and hilar lymphadenopathy: a prospective analysis. BMC Cancer 2011;11:100.

8 Sharples LD, et al: Clinical effectiveness and cost-effectiveness of endobronchial and endoscopic ultrasound relative to surgical staging in potentially resectable lung cancer: results from the ASTER randomised controlled trial. Health Technol Assess 2012;16:1-75, iii-iv.

9 Tanner NT, Pastis NJ, Silvestri GA: Training for linear endobronchial ultrasound among US pulmonary/critical care fellowships: a survey of fellowship directors. Chest 2013;143: 423-428.
10 Xiang Y, et al: EBUS-TBNA by a new Fuji EBUS scope (with video). J Thorac Dis 2013;5:36-39.

11 Ernst A, Silvestri GA, Johnstone D; American College of Chest Physicians: Interventional pulmonary procedures: guidelines from the American College of Chest Physicians. Chest 2003;123:1693-1717.

12 Medford AR: Learning curve for EBUSTBNA: longer than we may think. Respiration 2015;90:173.

13 Stather DR, et al: Endobronchial ultrasound learning curve in interventional pulmonary fellows. Respirology 2015;20:333-339.

14 Young A, Miller JP, Azarow K: Establishing learning curves for surgical residents using cumulative summation (CUSUM) analysis. Curr Surg 2005;62:330-334.

15 Rusch VW, et al: The IASLC lung cancer staging project: a proposal for a new international lymph node map in the forthcoming seventh edition of the TNM classification for lung cancer. J Thorac Oncol 2009;4:568-577.

$16 \mathrm{Hu} \mathrm{Y}$, et al: Attaining proficiency with endobronchial ultrasound-guided transbronchial needle aspiration. J Thorac Cardiovasc Surg 2013;146:1387-1392.e1.

17 Yasufuku K, Nakajima T, Fujiwara T, et al: Role of endobronchial ultrasound- guided transbronnchial needle aspiration in the management of lung cancer. Gen Thorac Cardiovasc Surg 2008;56:268-276.

18 Yasufuku K, Nakajima T, et al: Comparison of endobronchial ultrasound, positron emission tomography, and CT for lymph node staging of lung cancer. Chest 2006;130:710-718.

19 Agarwal R, Srinivasan A, Aggarwal AN, Gupta D: Efficacy and safety of convex probe EBUSTBNA in sarcoidosis: a systematic review and meta-analysis. Respir Med 2012;106:883-892.

20 Adams K, Shah PL, Edmonds L, Lim E: Test performance of endobronchial ultrasound and transbronchial needle aspiration biopsy for mediastinal staging in patients with lung cancer: systematic review and meta-analysis. Thorax 2009;64:757-762.

21 Stather DR, Maceachern P, Rimmer K, Hergott CA, Tremblay A: Validation of an endobronchial ultrasound simulator: differentiating operator skill level. Respiration 2011;81:325-332.

22 Konge L, et al: Simulator training for endobronchial ultrasound: a randomised controlled trial. Eur Respir J 2015;46:1140-1149.
23 Konge L, et al: Using virtual-reality simulation to assess performance in endobronchial ultrasound. Respiration 2013;86:59-65.

24 Huang CT, Chen CY, Ho CC, Yu CJ: A rare constellation of empyema, lung abscess, and mediastinal abscess as a complication of endobronchial-ultrasound-guided transbronchial needle aspiration. Eur J Cardiothorac Surg 2011;40:264-265.

25 von Bartheld MB, van Breda A, Annema JT: Complication rate of endosonography (endobronchial and endoscopic ultrasound): a systematic review. Respiration 2014;87:343-351.

26 Jeyabalan A, Medford AR: Endobronchial ultrasound-guided transbronchial needle aspiration: patient satisfaction under light conscious sedation. Respiration; 2014;88:244250.

27 Eapen GA, Shah AM, Lei X, et al; American College of Chest Physicians Quality Improvement Registry, Education: Complications, consequences, and practice patterns of endobronchial ultrasound-guided transbronchial needle aspiration: results of the AQuIRE registry. Chest 2013;143:1044-1053.

$28 \mathrm{Gu}$ P, et al: Endobronchial ultrasound-guided transbronchial needle aspiration for staging of lung cancer: a systematic review and metaanalysis. Eur J Cancer 2009;45:1389-1396.

29 Varela-Lema L, Fernandez-Villar A, RuanoRavina A: Effectiveness and safety of endobronchial ultrasound-transbronchial needle aspiration: a systematic review. Eur Respir J 2009;33:1156-1164.

30 Naik VN, Devito I, Halpem SH: Cusum analysis is a useful tool to assess resident proficiency at insertion of labour epidurals. Can J Anesth 2003;50:694-698.

31 Williams SM, Parry BR, Schlup MM: Quality control: an application of the cusum. BMJ 1992:304:1359-1361.

32 Abu-Hijleh M, El-Sameed Y, Eldridge K, et al: Linear probe endobronchial ultrasound bronchoscopy with guided transbronchial needle aspiration (EBUS-TBNA) in the evaluation of mediastinal and hilar pathology: introducing the procedure to a teaching institution. Lung 2013;191:109-115.

33 Bellinger CR, et al: Training in and experience with endobronchial ultrasound. Respiration 2014;88:478-483. 